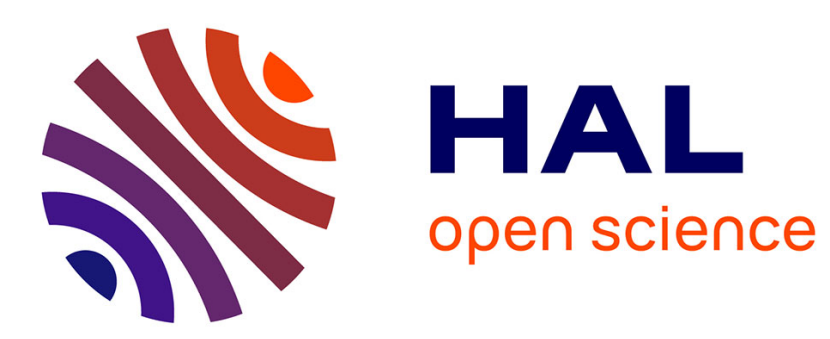

\title{
Modélisation de l'usinage formulée en Euler-Lagrange arbitraire
}

\author{
P. Joyot, R. Rakotomalala, M. Touratier
}

\section{To cite this version:}

P. Joyot, R. Rakotomalala, M. Touratier. Modélisation de l'usinage formulée en EulerLagrange arbitraire. Journal de Physique IV Proceedings, 1993, 03 (C7), pp.C7-1141-C7-1144. 10.1051/jp4:19937177 . jpa-00251808

\section{HAL Id: jpa-00251808 https://hal.science/jpa-00251808}

Submitted on 1 Jan 1993

HAL is a multi-disciplinary open access archive for the deposit and dissemination of scientific research documents, whether they are published or not. The documents may come from teaching and research institutions in France or abroad, or from public or private research centers.
L'archive ouverte pluridisciplinaire HAL, est destinée au dépôt et à la diffusion de documents scientifiques de niveau recherche, publiés ou non, émanant des établissements d'enseignement et de recherche français ou étrangers, des laboratoires publics ou privés. 


\title{
Modélisation de l'usinage formulée en Euler-Lagrange arbitraire
}

\author{
P. JOYOT, R. RAKOTOMALALA et M. TOURATIER*
}

Laboratoire Génie de Production, ENI de Tarbes, BP. 1629, Av. Azeirex, 65016 Tarbes cedex, France

${ }^{*}$ Laboratoire de Mécanique des Structures, ENSAM, 151 boulevard de l'Hôpital, 75013 Paris, France

\section{I - Introduction.}

Les modélisations numériques de l'enlèvement de matière sont généralement formulées suivant les approches classiques utilisées en simulation des procédés de mise en forme. L'approche Eulérienne est adoptée quand on s'intéresse uniquement au régime permanent établi (2) (3); toutefois, elle nécessite la connaissance de la géométrie "stationnaire" qui peut être obtenue au moyen d'un processus itératif (1). En formulation Lagrangienne, on peut étudier le régime transitoire, et l'histoire de la déformation du matériau peut être prise en compte de manière directe. La principale critique relative aux modèles basées sur cette approche est liée à la définition d'un critère de rupture qui influe de manière significative sur le champ des contraintes résiduelles (4). L'approche Euler-Lagrange Arbitraire (A.L.E) (5), basée sur la résolution des lois de conservation par rapport à un maillage arbitraire, est utilisée dans le modèle présenté dans cette étude. Cette formulation permet notamment de remédier aux problèmes de surface libre en approche Eulérienne, et à ceux liés aux fortes distorsions du maillage en appproche Lagrangienne. Le modèle porte sur la coupe orthogonale à copeau continu et à caractère stationnaire : on s'intéresse uniquement au régime permanent établi.

II - Equations "A.L.E" et discretisation explicite.

En description Euler-Lagrange Arbitraire, on définit deux mouvements distincts:

- le mouvement matériel, défini par : $\quad x=\varphi(X, t)$,

- et le mouvement arbitraire ou mouvement de grille donné par : $x=\Psi(\xi, t)$.

Ces deux lois de mouvement permettent de définir la vitesse matérielle $V=(\partial \varphi / \partial t)_{X}=$ cte et

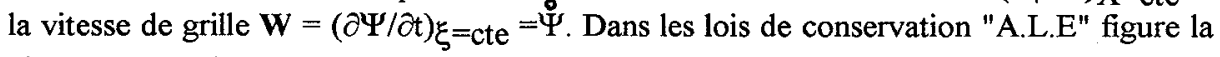
vitesse convective $\mathrm{C}=\mathrm{V}-\mathrm{W}$. La loi de conservation de la masse et de l'énergie, prise sous leur forme "intégrale" (1) et (2) sont discrétisées par une méthode des volumes finis. La loi de conservation de quantité de mouvement est discrétisée au moyen de la méthode des éléments finis avec une matrice de masse réduite, et permet d'obtenir l'équation matricielle (3):

$$
\begin{aligned}
& 0 \\
& \rho+\text { C. } \nabla \rho+\rho \operatorname{div} V=0 \\
& \frac{\partial}{\partial t} \int_{\omega(t)} \rho \cdot e \cdot d \omega+\int_{\Gamma(t)} \rho e C \cdot n d \Gamma=\int_{\omega(t)} \rho r d \omega+\int_{\omega(t)} \sigma \cdot \dot{\varepsilon} \cdot d \omega+\int_{\Gamma(t)} q \cdot n \cdot d \Gamma \\
& \mathbf{M}^{\mathrm{V}} \stackrel{0}{\mathbf{V}}+\mathbf{F}^{\mathrm{trm}}+\mathbf{F}^{\mathrm{int}}+\mathbf{F}^{\mathrm{ext}}+\mathbf{F}^{\mathrm{b}}+\mathbf{F}^{\mathrm{hgr}}=0
\end{aligned}
$$


L'intégration par rapport au temps est effectuée suivant un algorithme explicite et le calcul à chaque pas de temps est schématisé sur la figure 1 :



figure 1 : intégration explicite

III- Quelques précisions sur le modèle d'usinage.

Une loi de comportement thermoviscoplastique (Johnson-Cook) est adoptée pour représenter la rhéologie du matériau à usiner, et le contact outil-matière est modélisé au moyen de la loi de Coulomb. Une géométrie initiale arbitraire est introduite pour l'initialisation du calcul : sur les surface libres du copeau et de la pièce, les noeuds du maillage sont eulériens suivant la tangente à la surface et lagrangiens suivant la direction normale; les noeuds intérieurs au domaine sont de nature "A.L.E". Le calcul est arrêté lorsque les conditions de surface libre en régime stationnaire sont satisfaites (vitesses normales nulles sur les surfaces libres).

IV-Résultats de la simulation.

La simulation présentée a été éffectuée au moyen du code de calcul explicite Radioss, et concerne l'acier $42 \mathrm{CD} 4$ dans les conditions de coupe suivantes : -vitesse de coupe $(10 \mathrm{~m} / \mathrm{s})$; angle de coupe $\left(0^{\circ}\right)$; profondeur de coupe $(0.05 \mathrm{~mm})$. Le comportement du matériau est représentée par la loi de Johnson -Cook, généralement adoptée pour les problèmes à taux de cisaillement élevés et sensibles à la température. La figure 2 représente la géométrie initiale et la géométrie finale stabilisée avec le champ des vitesses correspondant. L'évolution des déplacements des noeuds à la surface libre est donnée sur la figure 3 et confirme l'évolution vers une solution stationnaire. La simulation permet aussi d'obtenir différentes grandeurs associées au régime permanent. La figure 4 représente le champ des 
contraintes équivalentes de Von Mises. La répartition des contraintes met en évidence une zone à cisaillement élevé (bande de cisaillement primaire). Sur la figure 5 est représenté le champ de température : la température maximale est de de l'ordre de $600^{\circ} \mathrm{C}$ et apparaît au voisinage de la surface de contact "outil-copeau". Ces différentes constatations sont convenables si l'on se réfère aux résultats numériques et expérimentaux disponibles dans la littérature.
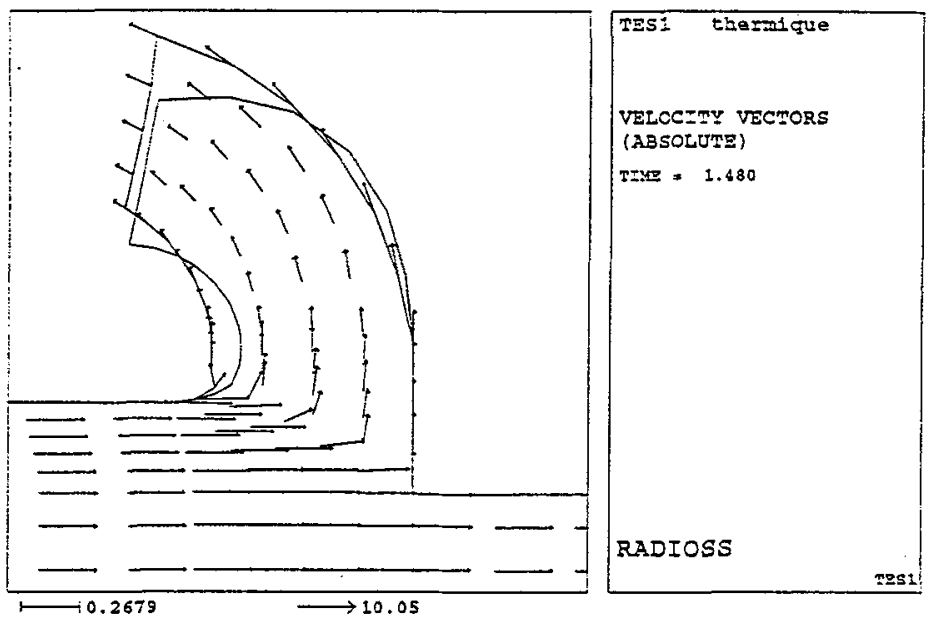

figure 2 : géométrie initiale- géométrie finale - champ des vitesses stationnaire

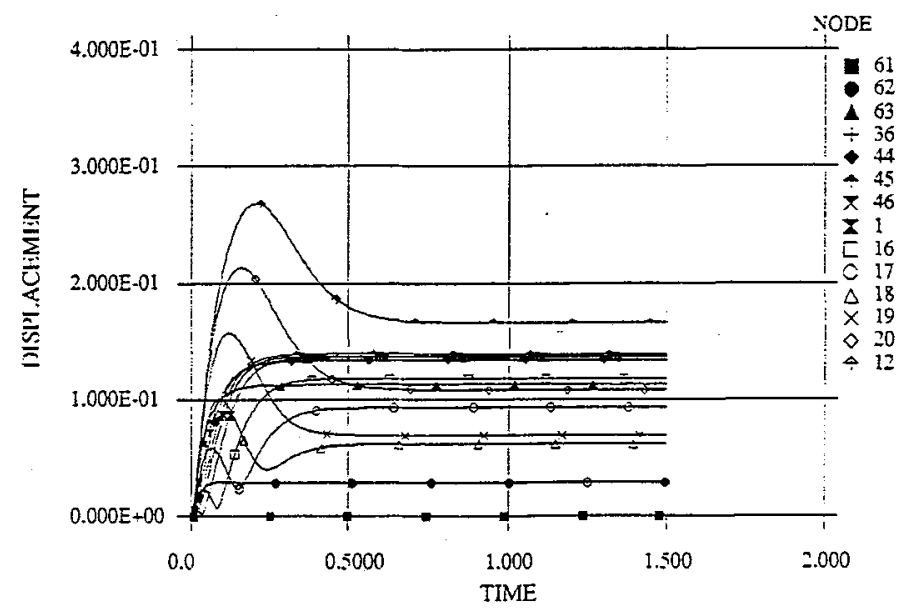

figure 3 : déplacement des noeuds de surface libre au cours du calcul 

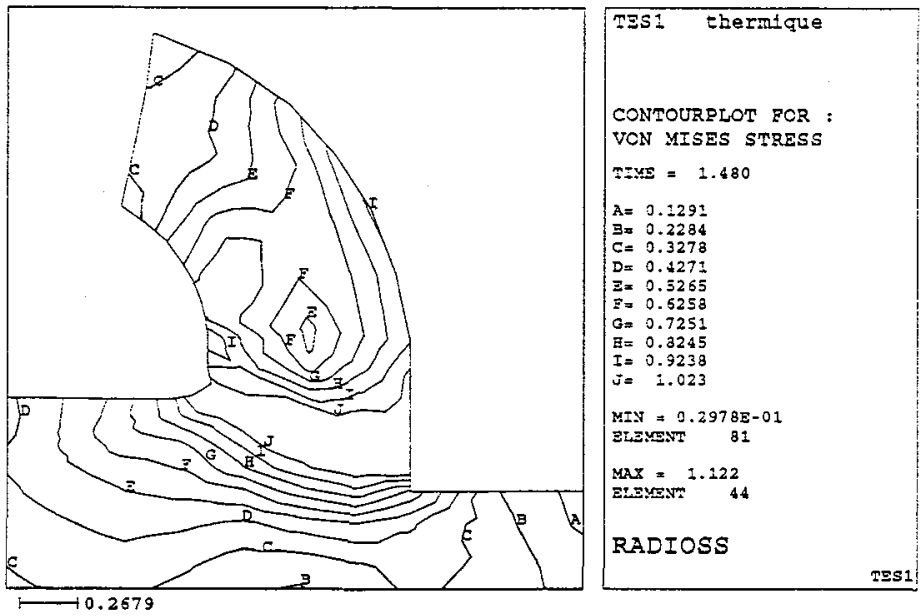

figure 4 : champ des contraintes de Von Mises (solution stationnaire)
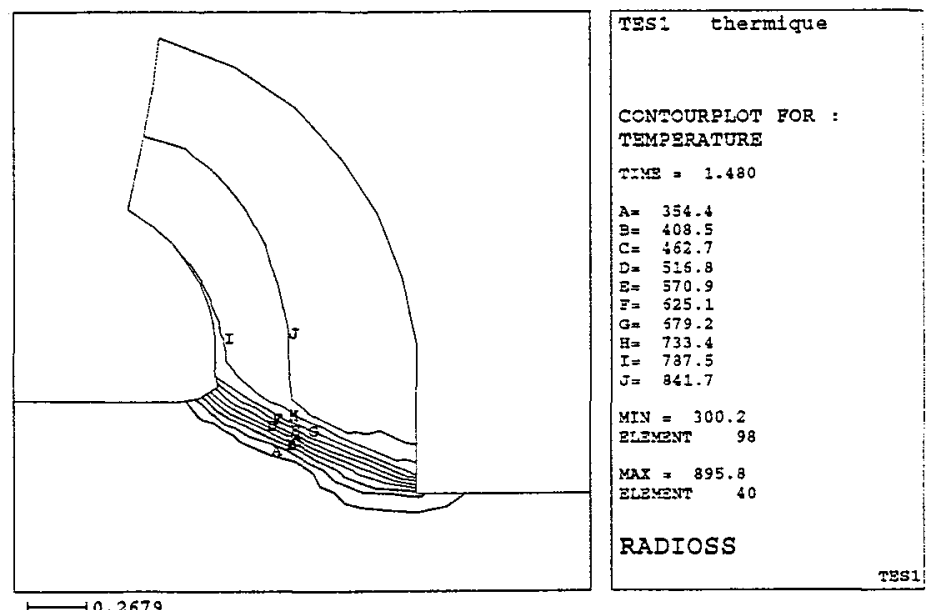

figure 5 : champ de Température (solution stationnaire)

1) O.C ZIEKIEWICZ, P.C. JAIN, O.C. ONATE, Int. J. Struct.., 14, 15-38, (1978)

2) J.S. STRENKOWSKI, K.J. MOON, Trans. of A.S.M.E., 112, 313-318, (1990)

3) P. GILORMINI, Thèse Doct.Ing., E.N.S.Mines Paris, (1982)

4) J.S. STRENKOWSKI, J.T. Caroll, Trans. of A.S.M.E., 107, 349-354, (1985)

5) J. HUENTINK, P.T. VREEDE, J. VAN DER LUGT, Int. J. Num.Meth. Engrg., 30, 1441-1457, (1990)

6) F.ARNEAUDAU, F.PERIE, G. WINKELMULLER, Séminaire Radioss, Sophia Antipolis, (1991) 